\title{
Synthesis of Cyclic Compounds Having exo-Methylene Groups through the Diels-Alder Reactions of Vinyl Allenes Obtained from Propargyl Bromide and Indium
}

\author{
Kooyeon Lee and Phil Ho Lee \\ Department of Chemistry and Institute for Basic Science, Kangwon National Lniversity, Chunchon 200-701, Korea \\ E-mail: phleeakangwon.ackr \\ Received Atrgust 10, 2007
}

Key Words : Diels-Alder reaction. Indium. exo-Methỵlene compound. Propargyl bromide. Vinỵl allene

Because allene is a very interesting compound having a hybrid character of C.C double and triple bond, vinyl allenes have been recognized as versatile building blocks in organic synthesis. ${ }^{1}$ In particular, vinyl allenes take part in not only the Diels-Alder reaction ${ }^{2}$ as the 1.3-diene moiety but also transition metal-catalyzed organic reactions, ${ }^{3}$ affording efficient synthetic methods for complex ring compounds. However. because it is not easy to effectively prepare a variety of vinyl allenes, its application to organic reactions has been linited despite the potential of vinyl allenes in organic synthesis. Although vinyl allenes were used in the DielsAlder reactions. development of synthetic method of cyclic compounds having exo-methy lene group is still required due to its utility in synthesis of natural products with biological activities. ${ }^{+}$Recently, we have demonstrated that allenylindiunss generated in situ from indium and propargyl bronides are effective cross-coupling partners in palladiumcatalyzed cross-coupling reactions to produce substituted allenes in excellent yields. " In continuation of our studies directed toward preparative method of vinyl allenes with allenyindiun. we describe herein the Diels-Alder reaction of vinyl allenes possessing 3.4- and 4,5-disubstituents and ketone group with a variety of dienophiles to give cyclic conpounds having exo-methylene group (Scheme 1)

First, 3-methyl-4-phenyl-1,2.4-pentatriene (1a) as vinyl allene was prepared from the reaction of $\alpha$-bromostyrene with allenylindium obtained from indiun and 1-bromo-2butyne and then. the Diels-Alder reactions with dienophiles were examined to obtain cyclic compounds having exomethylene group. The results are summarized in Table 1. Reaction of 1 a with maleic anhydride (2a) produced the Diels-Alder adduct $3 \mathrm{a}$ in $96 \%$ vield in toluene at $100^{\circ} \mathrm{C}$ for

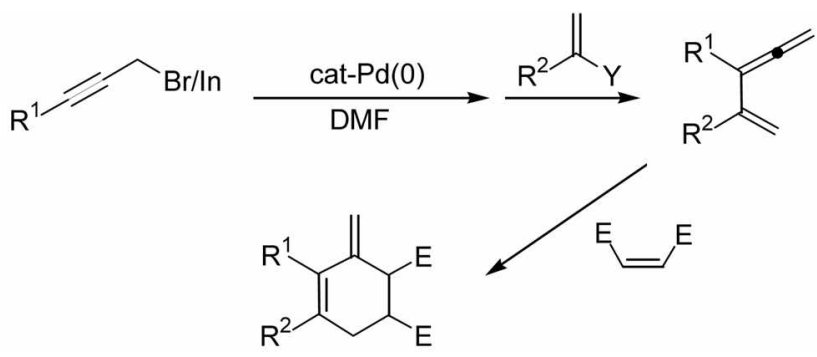

Scheme 1. Synthesis of cyclic compounds having exo-methylene group trom vinyl allenes and dienophiles.
3 h (entry 1). Also. 3-ethyl-4-phenyl-1.2,4-pentatriene (1b) reacted with a variety of dienophiles such as $2 \mathrm{a} . N$-phenyl maleinide (2b). dimethyl maleate (2c). and 1,4-naphthoquinone (2d), producing the desired products (3b, $\mathbf{3 c}$. $3 \mathbf{d}$, and $3 \mathrm{e}$ ) in good to excellent yields in toluene (entries 2-5). Stimulated by these results, tandem cross-coupling reaction of $\alpha$-bromostyrene with l-bromo-2-pentyne and indium (l equiv) in the presence of $\mathrm{Pd}\left(\mathrm{PPh}_{3}\right)_{4}(4 \mathrm{~mol} \%)$ in $\mathrm{DMF}$ followed by Diels-Alder reaction with $2 \mathrm{a}$ was attempted to obtain $3 \mathbf{b}$ in one-pot procedure. However. the desired product $3 \mathrm{~b}$ was produced in $7 \%$ yield in DMF. Although the cross-coupling product $\mathbf{1 b}$ was produced smootlly. the following Diels-Alder reaction did not proceed effectively Treatment of $\mathbf{1 b}$ with $\mathbf{2 a}$ did not proceed in THF and DMF In addition, hetero Diels-Alder reactions were tested Subjecting $\mathbf{1 b}$ to ethyl glyoxylate (2e) provided exclusively 3.6-dilydro-2 $\mathrm{H}$-pyran (3) having exo-metlyylene group in $80 \%$ yield (entry 6 ). No constitutional isomeric product is formed in this reaction. indicating that the electron-rich central carbon of vinyl allene preferentially adds to the more electron-deficient carbon of dienophile. Although compound $1 \mathrm{~b}$ reacted with ethyl acrylate (2i) to afford mixture of

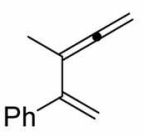

$1 \mathrm{a}$

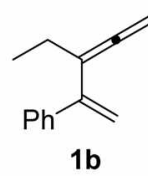

$1 \mathrm{~b}$

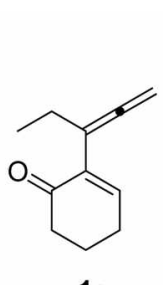

$1 c$

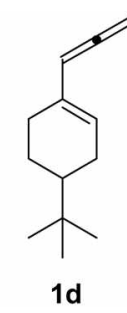

$1 d$

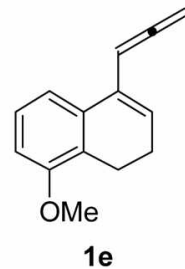

$1 e$
Figure 1. Vuyl allenes.<smiles>O=C1C=CC(=O)O1</smiles>

$2 a$<smiles>CCOC(=O)C=O</smiles>

$2 e$<smiles>O=C1C=CC(=O)N1c1ccccc1</smiles>

$2 b$<smiles>C=CC(=O)OCC</smiles>

$2 f$<smiles>COC(=O)/C=C\C(=O)OC</smiles>

2c<smiles>C#CC(=O)OCC</smiles>

$2 \mathrm{~g}$<smiles>O=C1C=CC(=O)c2ccccc21</smiles><smiles>COCC#CC(=O)OC</smiles>

Figure 2. Dienophiles. 
Table 1. $[4+2]$ Cycloaddition reaction of vinyl allenes with dienophiles ${ }^{i t}$

16

Reactions were carried out with finyl allene ( 1 equiv.) and dienophile ( 1 equiv.) unless otherwise noted. Reaction conditions: Toluene $\left(100^{\circ} \mathrm{C}\right)$ for entries $1-7$ and $11-12$. $\mathrm{CH}_{2} \mathrm{Cl}-\left(25^{\circ} \mathrm{C}\right)$ for entries 8 and $9 . \mathrm{CH}_{2} \mathrm{Cl}-\left(65^{\circ} \mathrm{C}\right)$ for entry 10. "Isolated yields. "Dienophile (3 equiv) was used. "Dienophile (30 equiv.) was used. 'Dienophile (5 equiv.) was used. ${ }^{\prime}$ Regioisomeric ratio. Ethyl 4-etlyl-5-methylene-3-phenylcyclohex-3-enecarbosylate was produced in major. ${ }^{8}$ Toluene $\left(25^{\circ} \mathrm{C}\right)$ was used. ${ }^{\circ} \mathrm{CH}_{2} \mathrm{Cl}_{2}\left(25^{\circ} \mathrm{C}\right)$ was used. 'Dienophile (2 equiv) was used. 'Diastereomeric ratio.<smiles>CCOC(=O)c1cc(C)c(CC)c(-c2ccccc2)c1</smiles>

4a $65 \%$<smiles>COC(=O)c1cc(-c2ccccc2)c(C)c(C)c1C(C)=O</smiles>

4b $82 \%$<smiles>CCc1c(C)c(C(C)=O)c(C(C)=O)c2c1C(=O)CCC2</smiles>

4c $95 \%$
Figure 3. Benzene derivatives from Diels-Alder reaction and aromatization.

regioisomeric products in $76 \%$ yield, ethyl 4-ethyl-5-methylene-3-phenylcyclohex-3-enecarboxylate was produced in major (entry 7). Although exposure of vinyl allene 1c to $2 a$ gave exo-methylenecyclohexene derivative $3 \mathbf{h}$ in $20 \%$ yield in toluene, adduct $3 \mathrm{~h}$ was produced in $77 \%$ yield in $\mathrm{CH}_{2} \mathrm{Cl}_{2}$ at room temperature for 13 h (entry 8 ). In the case of $\mathbf{2 b}$, $[4+2]$ cycloaddition adduct $3 \mathbf{i}$ was obtained in $83 \%$ yield in $\mathrm{CH}_{2} \mathrm{Cl}_{2}$ at room temperature for $13 \mathrm{~h}$ (entry 9). Reaction of 1c with $2 \mathrm{e}$ furnished pyran $3 \mathbf{j}$ having exo-methylene group in $87 \%$ yield with complete regioselectivity (entry 10). Vinyl allenes (1d and 1e) obtained from vinyl triflates and allenylindium were treated with $\mathbf{2} \mathbf{b}$ to produce the desired products (3k and 3l) in $92 \%(\mathrm{dr}=1: 1.5)$ and $74 \%$ yields, respectively (entries 11 and 12 ). Dienopliles having triple bond such as ethyl propiolate (2g) and DMAD (2h) were used to Diels-Alder reaction to give rise to multi-substituted benzene derivatives. Reaction of $1 \mathrm{~b}$ with $2 \mathrm{~g}$ produced selectively ethyl 4-etlyyl-3-methyl-5-phenyl benzoate ( + a) in $65 \%$ yield in toluene at $100^{\circ} \mathrm{C}$ for $20 \mathrm{~h}$. Compound $1 \mathrm{a}$ and 1c were treated with DMAD to afford th and $t \mathrm{c}$ in $82 \%$ (toluene, $100^{\circ} \mathrm{C} .4 \mathrm{~h}$ ) and $95 \%\left(\mathrm{CH}_{2} \mathrm{Cl}_{2} .65^{\circ} \mathrm{C} .15 \mathrm{~h}\right.$ ) yields, respectively. through Diels-Alder reaction followed by aromatization.

In summary. we have shown that cyclic compounds having exo-methylene group were selectively produced through the Diels-Alder reaction of vinyl allenes obtained from propargyl bromide and indium with a variety of symmetric and unsymmetric dienophiles in good to excellent yields. Also. dienophiles having triple bond gave multi-substituted benzene derivatives through Diels-Alder reaction followed by aromatization.

\section{Experimental Section}

5-Methyl-4-methylene-6-phenyl-3a,4,7,7a-tetrahydroisobenzofuran-1,3-dione (3a): A mixture of 3-methyl-4phenyl-1,2.4-pentatriene (1a) $(46.8 \mathrm{mg} .0 .3 \mathrm{mmol})$ and maleic anlydride $(29.4 \mathrm{mg} .0 .3 \mathrm{mmol})$ in toluene $(1.0 \mathrm{~mL})$ was heated at $100^{\circ} \mathrm{C}$ for $4 \mathrm{~h}$. The reaction mixture was cooling to room temperature and then quenched with $\mathrm{NaHCO}_{3}$ (sat. aq.). The aqueous layer was extracted with $\mathrm{CH}_{2} \mathrm{Cl}_{2}(20 \mathrm{~mL} \times 3)$ and the combined organics were washed with brine. dried with $\mathrm{MgSO}_{4}$ and concentrated. Recrystallization using methylene chloride and $n$-hexane gave 3a (74.0 mg. 96\%) as a white solid. 'H NMR (300 $\left.\mathrm{MHz}, \mathrm{CDCl}_{3} .25^{\circ} \mathrm{C}, \mathrm{TMS}\right) \delta 7.40-7.28$ (m. 3H). 7.18-7.15 $(\mathrm{m}, 2 \mathrm{H}) .5 .52(\mathrm{~s} . \mathrm{lH}), 5.47(\mathrm{~s}, \mathrm{lH}), 4.06(\mathrm{~d} . J=9.34 \mathrm{~Hz} . \mathrm{lH})$, 3.56 (ddd. $J=9.58 .6 .66 .3 .19 \mathrm{~Hz} .1 \mathrm{H}$ ). 2.94 (dd. $J=16.92$. 
$2.80 \mathrm{~Hz}, \mathrm{lH}) .2 .78-2.66(\mathrm{~m}, \mathrm{IH}), 1.82(\mathrm{~d} . J=1.54 \mathrm{~Hz} .3 \mathrm{H}):$ ${ }^{13} \mathrm{C}$ NMR $\left(75 \mathrm{MHz}, \mathrm{CDCl}_{3}\right) \delta 173.3 .171 .3,141.4 .135 .5$. 135.4. 129.6. 128.4. 128.1, 127.4. 116.2, 46.2, 40.0. 29.4. 17.0: IR (film) 2951. 1731. 1434. 1241. 1060.912.818.759 $\mathrm{cm}^{-1}$; m. p. 108-109 ${ }^{\circ} \mathrm{C}$; HRMS (EI) calcd for $\mathrm{C}_{16} \mathrm{H}_{1 \downarrow} \mathrm{O}_{3} \mathrm{M}^{+}$ 254.0943 . found 254.0945 .

5-Ethyl-4-methylene-6-phenyl-3a,4,7,7a-tetrahydroisobenzofuran-1,3-dione (3b): ${ }^{1} \mathrm{H}$ NMR $\left(400 \mathrm{MHz}, \mathrm{CDCl}_{3} .25\right.$ ${ }^{\circ} \mathrm{C}$. TMS) $\delta 7.38-7.34(\mathrm{~m}, 2 \mathrm{H}), 7.31-7.28(\mathrm{~m}, \mathrm{lH}) .7 .14-7.11$ $(\mathrm{m}, \mathrm{lH}) .5 .50(\mathrm{~d}, J=0.7 \mathrm{~Hz} .1 \mathrm{H}), 5.45$ (s. $1 \mathrm{H}), 4.04$ (d. $J=$ $9.51 \mathrm{~Hz} . \mathrm{IH}$ ). 3.56 (ddd. $J=9.39,6.56,2.70 \mathrm{~Hz}, \mathrm{lH}$ ), 2.87 (dd. $J=16.24,2.81 \mathrm{~Hz} .1 \mathrm{H}) .2 .67$ (ddd. $J=16.25,6.56 .2 .23$ $\mathrm{Hz}, \mathrm{lH}), 2.40-2.31$ (m. lH), 2.23-2.14 (m. lH). 0.88 (t, $J=$ $7.40 \mathrm{~Hz}, 3 \mathrm{H}):{ }^{13} \mathrm{C}$ NMR $\left(100 \mathrm{MHz} . \mathrm{CDCl}_{3}\right): \delta 173.5 .171 .3$. $141.3 .137 .0,135.0 .134 .4$. 128.5, 127.6, 127.3. 116.0, 47.7. 40.3, 29.8. 22.9. 13.3: IR (film) $1844,1781,1700.904 \mathrm{~cm}^{-1}$. m.p. $135-136^{\circ} \mathrm{C}$ : MS (EI) $m z 268\left(\mathrm{M}^{-}\right)$.

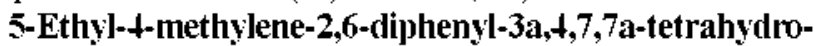
isoindole-1,3-dione (3c): ${ }^{1} \mathrm{H}$ NMR $\left(400 \mathrm{MHz} . \mathrm{CDCl}_{3}, 25\right.$ $\left.{ }^{\circ} \mathrm{C} . \mathrm{TMS}\right) \delta 7.48-7.45(\mathrm{~m}, 2 \mathrm{H}) .7 .40-7.32(\mathrm{~m} .3 \mathrm{H}), 7.27-7.24$ (m. 3H). 7.17-7.14 (m. 2H), 5.50 (s. $1 \mathrm{H}), 5.40$ (s. lH), 3.93 (d. $J=8.87 \mathrm{~Hz} . \mathrm{lH}$ ). $3.45-3.4 \mathrm{l}$ (m. $\mathrm{lH}$ ). 3.00 (dd, $J=15.73$. $2.27 \mathrm{~Hz} .1 \mathrm{H}$ ). 2.67 (ddd. $J=15.70 .6 .39 .2 .37 \mathrm{~Hz}, \mathrm{IH}) .2 .48$ 2.39 (n. lH), $2.26-2.17$ (m. IH). $0.85(\mathrm{t} . J=7.25 \mathrm{~Hz}, 3 \mathrm{H})$. ${ }^{13} \mathrm{C}$ NMR $\left(100 \mathrm{MHz} . \mathrm{CDCl}_{3}\right) \delta 178.5,176.3 .141 .7,137.2$. 137.1. 134.7. 132.1, 129.2, 128.6. 128.3, 127.9, 127.0. 126.3. 115.1. 48.4. 39.9. 30.4. 23.0, 13.6: IR (film) 1781 . 1715. 1381. $1175 \mathrm{~cm}^{-1}$ : m.p. $141-1+2{ }^{\circ} \mathrm{C}$; MS (EI) $m z 343$ $\left(\mathrm{M}^{+}\right)$.

Dimethyl 4-ethyl-3-methylene-5-phenyl-cyclohex-t-ene1,2-dicarboxlate (3d): ${ }^{1} \mathrm{H}$ NMR $\left(400 \mathrm{MHz}, \mathrm{CDCl}_{3} .25^{\circ} \mathrm{C}\right.$. TMS) $\delta 7.36-7.31(\mathrm{~m}, 2 \mathrm{H}) .7 .28-7.24(\mathrm{~m}, \mathrm{lH}), 7.15-7.12(\mathrm{~m}$. $2 \mathrm{H}), 5.30$ (s. IH). $5.23(\mathrm{~s}, 1 \mathrm{H}), 3.93(\mathrm{~d}, J=3.56 \mathrm{~Hz} . \mathrm{H})$. $3.72(\mathrm{~s}, 3 \mathrm{H}) .3 .69(\mathrm{~s}, 3 \mathrm{H}) .3 .05-2.94(\mathrm{~m} .2 \mathrm{H}), 2.69-2.58(\mathrm{~m}$. lH), 2.21-2.05 (m. $2 \mathrm{H}) .0 .91$ (t. $J=7.43 \mathrm{~Hz}, 3 \mathrm{H}) .{ }^{13} \mathrm{C}$ NMR $\left(100 \mathrm{MHz}, \mathrm{CDCl}_{3}\right) \delta$ 173.5. 172.1. 142.9, 139.5, 137.2 . 133.0. 128.3. 128.2. 127.7, 126.7. 113.9, 52.0, 48.6. 41.1. 32.3. 22.1. 13.7: IR (film) 2952, 1739, $1205 \mathrm{~cm}^{-1}$; MS (EI) $m=314\left(\mathrm{M}^{-}\right)$.

2-Ethyl-1-methylene-3-phenyl-1,4,4a,9a-tetrahydroanthraquinone (3e): ${ }^{1} \mathrm{H}$ NMR (400 $\mathrm{MHz} . \mathrm{CDCl}_{3,}, 25^{\circ} \mathrm{C}$. TMS) $\delta 8.10-8.07(\mathrm{~m} .1 \mathrm{H}) .8 .05-8.03(\mathrm{~m} .1 \mathrm{H}) .7 .78-7.72(\mathrm{~m}$. $2 \mathrm{H}), 7.38-7.33$ (m. $2 \mathrm{H}), 7.29-7.25$ (m. $3 \mathrm{H}), 5.3 \mathrm{l}(\mathrm{s}, \mathrm{lH})$. 4.88 (s. $1 \mathrm{H}$ ). 4.11 (dt. $J=3.61 .1 .41 \mathrm{~Hz}, 1 \mathrm{H}) .3 .58$ (q. $J=$ $5.56 \mathrm{~Hz}, 1 \mathrm{H}) .2 .95(\mathrm{dd} . J=18.08 .5 .97 \mathrm{~Hz}, 1 \mathrm{H}) 2.61(\mathrm{dd} . J=$ 18.06. $5.45 \mathrm{~Hz} .1 \mathrm{H}), 2.17-2.11(\mathrm{~m} .2 \mathrm{H}), 0.92$ (t. $J=7.45 \mathrm{~Hz}$. $3 \mathrm{H}):{ }^{13} \mathrm{C}$ NMR $\left(100 \mathrm{MHz}, \mathrm{CDCl}_{3}\right) \delta 197.0 .196 .9 .142 .9$. 137.1. 136.4. 135.1, 134.4, 134.3. 134.1, 133.8, 128.3. 127.7. 126.9, 126.8, 127.7, 113.7, 54.8, 47.8, 32.8, 22.1 . 140: IR (film) $3060,1695,1594.703 \mathrm{~cm}^{-1}$; nt.p. 101-102 ${ }^{\circ} \mathrm{C}$ : MS (EI) $m z 328\left(\mathrm{M}^{+}\right)$.

Ethyl t-ethyl-3-methylene-5-phenyl-3,6-dihydro- $2 \mathrm{H}$ pyran-2-carboxylate (3f): ${ }^{1} \mathrm{H}$ NMR $\left(400 \mathrm{MHz}, \mathrm{CDCl}_{3}, 25\right.$ ${ }^{\circ} \mathrm{C}$. TMS) $87.38-7.28$ (m, 3H). 7.18-7.15 (m, 2H). 5.32 (s. lH), 5.15 (s. IH), 4.88 (s. IH). 4.71 (d. $J=17.13 \mathrm{~Hz} . \mathrm{lH})$. 4.37 (d. $J=17.13 \mathrm{~Hz}, 1 \mathrm{H}) .4 .28$ (q. $J=7.11 \mathrm{~Hz}, 2 \mathrm{H}) .2 .19$ (q. $J=7.29 \mathrm{~Hz}, 2 \mathrm{H}$ ), 1.32 (t. $J=7.17 \mathrm{~Hz}, 3 \mathrm{H}$ ). 0.97 (t. $J=$
$7.47 \mathrm{~Hz}, 3 \mathrm{H}),{ }^{12} \mathrm{C}$ NMR $\left(100 \mathrm{MHz} . \mathrm{CDCl}_{3}\right) \delta 170.5,138.1$, $136.0,135.6,131.3,128.4,128.3,127.5,111.1 .68 .0,61.2$, 21.2, 14.3. 13.8: IR (film) 2975, 1739. 1609. 1465, 1130 $\mathrm{cm}^{-1} ; \mathrm{MS}(\mathrm{EI}) m z 199\left(\mathrm{M}^{+}-\mathrm{CO}_{2} \mathrm{Et}\right)$.

Ethyl 4-ethyl-5-methylene-3-phenylcyclohex-3-enecarboxylate (3g, major compound): ${ }^{1} \mathrm{H}$ NMR $(400 \mathrm{MHz}$, $\left.\mathrm{CDCl}_{3} .25^{\circ} \mathrm{C}, \mathrm{TMS}\right) \delta 7.35(\mathrm{~m}, 2 \mathrm{H}), 7.27(\mathrm{~m} . \mathrm{lH}), 7.16(\mathrm{~d} . J$ $=6.83 \mathrm{~Hz} \cdot 2 \mathrm{H}), 5.09(\mathrm{~s}, \mathrm{lH}) .4 .97(\mathrm{~s}, \mathrm{lH}) .4 .14$ (q. $J=7.11$ Hz. $2 \mathrm{H}$ ). $2.83-2.77$ (m, lH). $2.7 \mathrm{l}$ (dd. $J=14.1,3.46 \mathrm{~Hz}, \mathrm{lH}$ ), 2.65-2.54 (m, 3H), $2.18-2.07$ (m. $2 \mathrm{H}$ ). 1.25 (t. $J=7.16 \mathrm{~Hz}$, $3 \mathrm{H}) .0 .93(\mathrm{t}, J=7.4 \mathrm{~Hz} \cdot 3 \mathrm{H}) ;{ }^{13} \mathrm{C}$ NMR $\left(100 \mathrm{MHz} . \mathrm{CDCl}_{3}\right) \delta$ $174.8,143.4,141.0 .136 .6 .134 .6,128.2,127.7 .126 .6$. $110.1,60.4 .40 .2,35.8 .35 .1 .21 .9,14.3 .14 .2$ : IR (film) 2932. 1732, 1442. $1178,1038,884,761,701 \mathrm{~cm}^{-1}$; MS (EI) $m \geq 270\left(\mathrm{M}^{-}\right)$.

Ethyl 3-ethyl-2-methylene-4-phenylcyclohex-3-enecarboxylate (3g, minor compound): ${ }^{1} \mathrm{H}$ NMR $(400 \mathrm{MHz}$, $\left.\mathrm{CDCl}_{3} .25^{\circ} \mathrm{C}, \mathrm{TMS}\right) \delta 7.33$ (t. $\left.J=7.38 \mathrm{~Hz} .2 \mathrm{H}\right), 7.24(\mathrm{~m}$, lH). $7.12(\mathrm{~m}, 2 \mathrm{H}), 5.22(\mathrm{~s}, \mathrm{lH}), 4.98(\mathrm{~s}, \mathrm{lH}), 4.19(\mathrm{~m} .2 \mathrm{H})$, $3.40($ t. $J=5.18 \mathrm{~Hz} . \mathrm{lH}) .2 .49(\mathrm{~m}, \mathrm{lH}), 2.33(\mathrm{dt} . J=18.4$, $5.20 \mathrm{~Hz}, \mathrm{lH}), 2.25 .2 .12(\mathrm{~m} .3 \mathrm{H}), 1.98(\mathrm{~m} . \mathrm{lH}) .1 .28(\mathrm{t}, J=$ $7.10 \mathrm{~Hz} .3 \mathrm{H}), 0.95$ (t. $J=7.44 \mathrm{~Hz}, 3 \mathrm{H}) ;{ }^{13} \mathrm{C}$ NMR $(100$ $\left.\mathrm{MHz}, \mathrm{CDCl}_{3}\right) \delta 173.9,143.8 .140 .5,137.9 .133 .7,128.1$, $127.6,126.4,111.1 .60 .4,47.5,31.1,25.7,22.2$. 14.3. 13.9; IR (film) 2931, 1732. 1605. 1443, 1373. 1308, 1258, 1155 . 1040. $759 \mathrm{~cm}^{-1}$ : MS (EI) $m \approx 270\left(\mathrm{M}^{+}\right)$.

5-Ethyl-t-methylene-3a,7,8,9,9a,9b-hexahydro-4H naphtho[1,2-c]furan-1,3,6-trione (3h): ${ }^{1} \mathrm{H}$ NMR $(400$ $\mathrm{MHz}, \mathrm{CDCl}, 25^{\circ} \mathrm{C}$. TMS) $\delta 5.67$ (s. $\left.1 \mathrm{H}\right), 5.63$ (s. IH). 4.02 (d. $J=9.52 \mathrm{~Hz}, \mathrm{lH}) .3 .46(\mathrm{dd}, J=9.50,5.15 \mathrm{~Hz}, 1 \mathrm{H}) .3 .15$ (octet, $J=7.27 .12 .02,14.68 \mathrm{~Hz} . \mathrm{lH}$ ). $2.68-2.62(\mathrm{~m} . \mathrm{lH}$ ), 2.57-2.48 (m, lH), 2.46-2.35 (m, lH), 2.33-2.03 (m. $2 \mathrm{H}$ ), $2.09-1.97$ (m, 2H), $1.76-1.63$ (m. lH). 1.00 (t. $J=7.39 \mathrm{~Hz}$. $3 \mathrm{H}) ;{ }^{13} \mathrm{C}$ NMR (100 MHz. $\left.\mathrm{CDCl}_{3}\right) \delta 199.2,171.0,170.4$, $153.3,137.6 .131 .2 .121 .0 .49 .7,44.7 .40 .0,37.2 .25 .8 .25 .3$, 21.2. 13.1: IR (film) $1885.1780,1662,1549,1200 \mathrm{~cm}^{-1}$. m.p. $156^{\circ} \mathrm{C}$; MS (EI) $m \geq 260\left(\mathrm{M}^{+}\right)$.

5-Ethyl-4-methylene-2-phenyl-3a,7,8,9,9a,9b-hexahydro$4 H$-benzo $[e]$ isoindole-1,3,6-trione (3i): ${ }^{1} \mathrm{H}$ NMR $(400$ $\left.\mathrm{MHz}, \mathrm{CDCl}_{3} .25^{\circ} \mathrm{C}, \mathrm{TMS}\right) \delta 7.65-7.32(\mathrm{~m}, 3 \mathrm{H}) .7 .15-7.13$ (m. 2H). 5.63 (s. 1H), 5.62 (s. 1H). $3.90(\mathrm{~d} . J=9.04 \mathrm{~Hz} .1 \mathrm{H})$. $3.46($ dd $J=4.92 .8 .87 \mathrm{~Hz}, 1 \mathrm{H}), 3.19$ (octet. $J=7.29 .11 .72$. $14.63 \mathrm{~Hz}, 1 \mathrm{H}) .2 .72-2.67(\mathrm{~m} .1 \mathrm{H}), 2.54-2.33(\mathrm{~m}, 3 \mathrm{H}), 2.28-$ $2.17(\mathrm{~m} .1 \mathrm{H}) .2 .08-1.99(\mathrm{~m} .2 \mathrm{H}) .1 .76-1.62(\mathrm{~m} . \mathrm{lH}) .1 .00(\mathrm{t}$. $J=7.33 \mathrm{~Hz}, 3 \mathrm{H}):{ }^{13} \mathrm{C}$ NMR $\left(100 \mathrm{MHz} . \mathrm{CDCl}_{3}\right): \delta 199.9$, $176.4,175.0,153.5 .139 .7,131.7,131.1,129.2 .128 .7$. 126.4. 120.0, 49.9. 43.7. 40.0, 38.2. 25.8, 25.5. 21.4. 13.4: IR (film) 1713. $1668.1498 \mathrm{~cm}^{-1}$ : m.p. 160-161 ${ }^{\circ} \mathrm{C}$ : MS (EI) $m: 335\left(\mathrm{M}^{-}\right)$

Ethyl 4-ethyl-3-methylene-5-oxo-3,5,6,7,8,8a-hexahydro$2 \boldsymbol{H}$-chromene-2-carboxylate (3j): ${ }^{1} \mathrm{H}$ NMR $(400 \mathrm{MHz}$, $\mathrm{CDCl}_{3} .25^{\circ} \mathrm{C}$. TMS) $\delta 5.56(\mathrm{~s} .1 \mathrm{H}), 5.27(\mathrm{~s}, \mathrm{lH}), 4.74(\mathrm{t} . J=$ $1.53 \mathrm{~Hz}, \mathrm{LH}$ ). $4.42-4.30$ (m. 3H). $2.59-2.28$ (m. $5 \mathrm{H}) .2 .07-$ $2.00(\mathrm{~m}, \mathrm{lH}) .1 .92-1.82(\mathrm{~m} . \mathrm{lH}) .1 .75-1.63(\mathrm{~m} . \mathrm{lH}) .1 .35(\mathrm{t}$. $J=7.10 \mathrm{~Hz}, 3 \mathrm{H}), 1.13(\mathrm{t} . J=7.45 \mathrm{~Hz}, 3 \mathrm{H}):{ }^{13} \mathrm{C}$ NMR $(100$ $\left.\mathrm{MHz}, \mathrm{CDCl}_{3}\right) \delta 201.8,169.2,142.6 .137 .3,135.4,114.1$, $76.8,61.5,42.7,31.5 .21 .8$. 19.2. 14.4. 14.2: IR (film) 2940 , 
1735. 1685, 1457, $1192 \mathrm{~cm}^{-1}$; MS (EI) $m z 264\left(\mathrm{M}^{+}\right)$

8-tert-Butyl-4-methylene-2-phenyl-3a, $4,6,7,8,9,9 \mathrm{a}, 9 \mathrm{~b}$ octahydrobenzo[e]isoindole-1,3-dione (3k, major compound): ${ }^{1} \mathrm{H} \mathrm{NMR}\left(400 \mathrm{MHz}, \mathrm{CDCl}_{3} .25^{\circ} \mathrm{C}\right.$, TMS) $\delta 7.44$ (t. $J=7.70 \mathrm{~Hz}, 2 \mathrm{H}) .7 .36(\mathrm{t}, J=7.47 \mathrm{~Hz} . \mathrm{lH}), 7.23(\mathrm{~d} . J=7.56$ $\mathrm{Hz}, 2 \mathrm{H}) .6 .04$ (s. IH). $5.30(\mathrm{~s}, 1 \mathrm{H}), 5.17(\mathrm{~s}, 1 \mathrm{H}), 3.80$ (d, $J=$ $8.30 \mathrm{~Hz}, \mathrm{lH}) .3 .36(\mathrm{dd}, J=8.0 \mathrm{l} .6 .86 \mathrm{~Hz}, \mathrm{lH}), 2.77(\mathrm{~m}, \mathrm{lH})$. $2.37(\mathrm{~m}, \mathrm{lH}) .2 .25(\mathrm{~m}, \mathrm{IH}) .2 .02(\mathrm{td} . J=12.65,8.87 \mathrm{~Hz}$. 1H), 1.73 (m. $2 \mathrm{H}) .1 .52(\mathrm{~m}, \mathrm{IH}), 1.32$ (qd, $J=12.48 .5 .41$ $\mathrm{Hz}, \mathrm{IH}):{ }^{13} \mathrm{C}$ NMR $\left(100 \mathrm{MHz}, \mathrm{CDCl}_{3}\right) \& 175.8,175.0$. 142.1. 133.0, 130.9. 128.0. 127.4, 125.3, 121.1. 113.5, 43.5. $42.2,41.4,32.3$. 31.1. 30.1. 26.0, 24.8, 21.5; IR (filnt) 3463 . 2959. 2867, 2249, 1775. $1709 \mathrm{~cm}^{-1}$ : MS (EI) $m: 349\left(\mathrm{M}^{+}\right)$.

4-Methoxy-12-methylene-16-phenyl-6,7,8,12,13,1+-hexahydro-16-aza-cyclopenta[a]phenanthrene-15,17-dione (31): ${ }^{1} \mathrm{H}$ NMR (400 MHz, $\mathrm{CDCl}_{3}, 25^{\circ} \mathrm{C}$, TMS) $\delta 7.41$ (m, 2H). 7.33 (n. lH). 7.23 (m, 3H), 7.14 (t. $J=8.05 \mathrm{~Hz}, 1 \mathrm{H}), 6.85$ (d, $J=2.25 \mathrm{~Hz} . \mathrm{IH}) .6 .75$ (d. $J=8.02 \mathrm{~Hz}, 1 \mathrm{H}), 5.52$ (s. IH). 5.35 (s. $1 \mathrm{H}$ ), 3.92 (d. $J=8.09 \mathrm{~Hz} .1 \mathrm{H}), 3.83$ (s, 3H), 3.50 (dd. $J=8.07,5.44 \mathrm{~Hz}, \mathrm{IH}) .3 .27(\mathrm{td} . J=16.33 .3 .32 \mathrm{~Hz}$. lH), $2.88(\mathrm{~m}, \mathrm{lH}) .2 .55$ (qd, $J=13.06,3.81 \mathrm{~Hz}, \mathrm{IH}$ ). 2.33 (m, IH), 2.13 (m. IH): ${ }^{13} \mathrm{C}$ NMR $\left(100 \mathrm{MHz}, \mathrm{CDCl}_{3}\right) \delta$

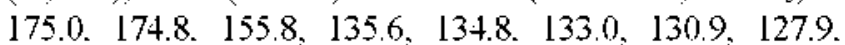
127.3. 126.4, 125.5, 125.3. 121.1. 116.1. 114.7. 108.1, 54.5. $45.0,43.3,34.3,23.0 .21 .8$. IR (film) $2834,1710,1572$. 1499, 1387. $126 \mathrm{I} \mathrm{cm}^{-1}$; m.p. $189-190^{\circ} \mathrm{C}$ : MS (EI) $m z 371$ $\left(\mathrm{M}^{+}\right)$.

Ethyl t-ethyl-5-methyl-3-phenyl benzoate (4a): ${ }^{1} \mathrm{H}$ NMR (400 MHz. CDCl, $25^{\circ} \mathrm{C}$. TMS) $\delta 7.84$ (d, $J=1.33$ $\mathrm{Hz}, 1 \mathrm{H}), 7.70(\mathrm{~d}, J=1.69 \mathrm{~Hz}, \mathrm{lH}) .7 .46-7.37(\mathrm{~m}, 3 \mathrm{H}), 7.30-$ $7.27(\mathrm{~m}, 2 \mathrm{H}), 4.35(\mathrm{q} . J=7.09 \mathrm{~Hz} .2 \mathrm{H}) .2 .60(\mathrm{q} . J=7.52 \mathrm{~Hz}$. $2 \mathrm{H}), 2.44(\mathrm{~s} .3 \mathrm{H}), 1.36(\mathrm{t}, J=7.18 \mathrm{~Hz} .3 \mathrm{H}), 0.99(\mathrm{t}, J=7.5 \mathrm{l}$ $\mathrm{Hz}, 3 \mathrm{H}):{ }^{13} \mathrm{C}$ NMR $\left(100 \mathrm{MHz}, \mathrm{CDCl}_{3}\right) \delta 166.8,145.6$. 142.4. 141.8. 136.6, 130.5, 129.2. 129.0, 128.0, 127.3 . 127.0. 60.8. 23.3, 19.7. 14.4. 14.1: IR (filnt) 2971. 2930 , $1717,1223 \mathrm{~cm}^{-1}$ : MS (EI) $m=268\left(\mathrm{M}^{+}\right)$.

Dimethyl 3,4-dimethyl-5-phenylphthalate (4b): A mixture of 3-methyl-4-phenyl-1.2.4-pentatriene (1a) (46.86 mg. $0.3 \mathrm{mmol}$ ) and DMAD (42.63 $\mathrm{mg} .0 .3 \mathrm{mmol}$ ) was heated in toluene $(1.0 \mathrm{~mL})$ at $100^{\circ} \mathrm{C}$ for $4 \mathrm{~h}$. The reaction misture was quenched with $\mathrm{NaHCO}_{3}$ (sat. aq.). The aqueous layer was extracted with $\mathrm{CH}_{2} \mathrm{Cl}_{2}(20 \mathrm{~mL} \times 3)$ and the combined organics were washed with water and brine, dried over $\mathrm{MgSO}_{4}$, filtered. and concentrated under reduced pressure. It was purified by recrystallization $\left(\mathrm{CH}_{2} \mathrm{Cl}_{3}\right.$ Hex) to give $\mathbf{4 b}$ (73.0 mg. 82\%). ${ }^{1} \mathrm{H}$ NMR (300 MHz, CDCl, $25^{\circ} \mathrm{C}$. TMS) $\delta$ 7.78 (s, IH). 7.47-7.36 (m. 3H), 7.28-7.25 (n, 2H), 3.99 (s. $3 \mathrm{H}), 3.86(\mathrm{~s} .3 \mathrm{H}) .2 .30(\mathrm{~s}, 3 \mathrm{H}) .2 .2 \mathrm{l}(\mathrm{s} .3 \mathrm{H}):{ }^{13} \mathrm{C}$ NMR $(100$ $\left.\mathrm{MHz} . \mathrm{CDCl}_{3}\right) \delta$ 170.5. 166.1, 143.1. 141.0, 140.4, 134.6. 134.4. 129.2. 129.1, 128.2, 127.3, 124.3. 52.6, 52.3. 17.8.
17.1: IR (film) 1730. 1432, $1323 \mathrm{~cm}^{-1}$; m.p. 107-108 ${ }^{\circ} \mathrm{C}$; MS (EI) $m \approx 298\left(\mathrm{M}^{+}\right)$.

Dimethyl 4-ethyl-3-methyl-5-ox0-5,6,7,8-tetrahydronaphthalene-1,2-dicarboxylate (4c): ${ }^{1} \mathrm{H}$ NMR (400 MHz, $\mathrm{CDCl}_{3} .25^{\circ} \mathrm{C}$, TMS) $\delta 3.90(\mathrm{~s} .3 \mathrm{H}), 3.87(\mathrm{~s}, 3 \mathrm{H}) .2 .99$ (q. $J=$ $7.34 \mathrm{~Hz}, 2 \mathrm{H}) .2 .96$ (t. $J=6.26 \mathrm{~Hz} .2 \mathrm{H}) .2 .67(\mathrm{t} . J=6.75 \mathrm{~Hz}$, $2 \mathrm{H}) .2 .33(\mathrm{~s}, 3 \mathrm{H}), 2.04$ (quintet, $J=6.50 \mathrm{~Hz}, 2 \mathrm{H}), 1.20(\mathrm{t} . J=$ $7.33 \mathrm{~Hz} .3 \mathrm{H}$ ): ${ }^{13} \mathrm{C}$ NMR (100 MHz. $\left.\mathrm{CDCl}_{3}\right) \delta 200.1,169.0$. 168.1. 148.4. 141.1. 136.4. 133.7. 133.4, 128.5, 52.6. 52.5. 40.7. 28.1, 23.9. 22.3. 15.6, 14.1; IR (film) 2953, 2359. 1739. $1690.1436 \mathrm{~cm}^{-1}$. MS (EI) $m z 304\left(\mathrm{M}^{-}\right)$

Acknowledgment. This work was supported by KOSEF through the National Research Lab. Program funded by the Ministry of Science and Teclunology (No. M1060000020306J0000-20310). by the Korea Science and Engineering Foundation (KOSEF, R01-2006-000-11283-0), and by the CMDS at KAIST. The NMR and mass data were obtained from the central instrumental facility in Kangwon National University

\section{References}

1. (a) Patai, S.. Ed. The Chemisoy of Ketenes. Allenes and Related Compounds: Wiley: New York. 1980. (b) The Chentsm of the Allenes: Landor. S. R.. Ed.: Academic: London1. 1982. (c) Smadja. W. Chem. Rev. 1983. 83. 263. (d) Schuster. H. F.: Coppola. G. M. Allenes in Organic Sunthesis' Wiley: New York, 1984. (e) Zimmer. R; Dinesh, C. U.; Nandanan, E; Khan, F. A. Chem. Rev. 2000. 100, 3067. (f) Modern Allene Chemison. Krause, N.. Hashmi. A. S. K. Eds.: Wiley-VCH: Weinheim. 2004

2. (a) Angelov. C. M.: Mondeshka. D. M.: Tancheva. I. N. Chen. Conmmm. 1985. 647. (b) Reich. H. T.: Eisenhart. E. K.: Whipple. W. L.; Kelly. M. J. J. Am. Chem. Soc, 1988, 110. 6432. (c) Dulcere J. P. Agati. V.: Faure. R. Chem. Commm. 1993. 270. (d) Spino, C.: Thibault C.: Gingras, S. J. Org. Chem 1998, 63, 5283. (e) Regás. D.: Afonso. M. M.: Rodríguez. M. L.: Palenzuela. J. A. J. Ong. Chem. 2003. 68. 7845. (f) Regás. D.: Ruiz. J. M.: A fontso. M. M.: Palenzuela. J. A. J. Ong. Chent. 2006. 71.9153.

3. (a) Murakami, M.: Itami. K.: Ito, Y. Angew. Chem. Int. Ed. 1995. 34. 2691. (b) Murakami. M.: Itami. K. Ito. Y. J. Am. Chem. Soc 1997. 119,7163 . (c) Lee. P. H. Lee. K. Angew. Chem. Int. Ed. 2005. H. 3253. (d) Lee. P. H.: Lee. K.: Kang. Y. J. Am. Chent. Soc. 2006. 128. 1139. (e) Funami. H.: Kusama. H.: Iwasawa. N. Angew. Chem. Int. Ed. 2007. 46.909.

4. (a) Cavallito, C. J; Fruehauf. D. M; Bailey: J. H. J. Am. Chem. Soc. 1948, 70, 3724. (b) Lee, K. H; Hail, I. H.: Mar, E. C. Starnes, C. O.; El Gebaly. S. A.: Waddell, T. G.: Hadgraft, R. I: Rufťner. C. G.: Weidner. I. Science 1977. 196. 533. (c) Spring. O.: Albert. K.: Gradmann. W. Phrochemistry 1981. 20. 1883. (d) Park. B. K.: Nakagawa. M.: Hirota. A.: Nakayama. M J. Antibiot. 1988. 41,751 . (e) Liu. S.: Liu. H.: Yan. W. Zhang. L.: Bai, N.: Ho. C.-T. Bioorg Hed Chem Lett 2004, 1t, 1101.

5. Lee. K.: Seomoon, D; Lee. P. H. Angew. Chem. Int Ed. 2002, H. 3901. 